OPEN ACCESS

Edited by:

Chao Yan,

East China Normal University,

China

Reviewed by:

Teresa Sanchez-Gutierrez,

Universidad Internacional

De La Rioja, Spain

Yuan Cao,

The Hong Kong Polytechnic

University, Hong Kong

${ }^{*}$ Correspondence:

Jin-feng Wen

jfwen2000@126.com

Lai-quan Zou

zoulq@smu.edu.cn

tThese authors have contributed

equally to this work

Specialty section:

This article was submitted to

Schizophrenia,

a section of the journal

Frontiers in Psychiatry

Received: 27 September 2019 Accepted: 11 May 2020

Published: 04 June 2020

Citation:

Li Z-I, Huang G-j, Li Z-t, Li S-b,

Wang $Y$-l, Zhao J-b, Wen J-f,

Hummel T and Zou L-q (2020) Chemosensory Anhedonia in Patients

With Schizophrenia and

Individuals With Schizotypy: A

Questionnaire Study.

Front. Psychiatry 11:481

doi: 10.3389/fpsyt.2020.00481

\section{Chemosensory Anhedonia in Patients With Schizophrenia and Individuals With Schizotypy: A Questionnaire Study}

\author{
Zi-lin $\mathrm{Li}^{1+}$, Gao-jie Huang ${ }^{1+}$, Ze-tian $\mathrm{Li}^{1}$, Shu-bin $\mathrm{Li}^{1}$, Yi-le Wang ${ }^{1}$, Jiu-bo Zhao ${ }^{1}$, \\ Jin-feng Wen ${ }^{2 *}$, Thomas Hummel ${ }^{3}$ and Lai-quan Zou ${ }^{1,3,4^{*}}$ \\ ${ }^{1}$ Chemical Senses and Mental Health Lab, Department of Psychology, School of Public Health, Southern Medical University \\ (Guangdong Provincial Key Laboratory of Tropical Disease Research), Guangzhou, China, 2 Department of Psychology, \\ Guangdong 999 Brain Hospital, Guangzhou, China, ${ }^{3}$ Smell and Taste Clinic, Department of Otorhinolaryngology, Technische \\ Universität Dresden, Dresden, Germany, ${ }^{4}$ Department of Psychiatry, Zhujiang Hospital, Southern Medical University, \\ Guangzhou, China
}

Anhedonia, the loss or decline of the ability to enjoy pleasure, is an important clinical characteristic of schizophrenia. Schizotypal traits refer to the appearance of subclinical symptoms of schizophrenia across normal people. Still, few studies have investigated chemosensory anhedonia in schizophrenia patients and schizotypy individuals. Seventyone schizophrenia patients (SCZ), 162 schizotypy individuals (SCT) as selected by the Schizotypal Personality Questionnaire (SPQ), and 182 healthy controls $(\mathrm{HC})$ participated in our study. We used the Positive and Negative Syndrome Scale (PANSS) to measure the clinical symptoms of schizophrenia patients. All participants completed the Chemosensory Pleasure Scale (CPS), which was used to assess participants' smell and taste hedonic capacities. We found that the three groups differed in chemosensory anhedonia. The SCZ group presented more severe chemosensory anhedonia than the SCT group, and the SCT group presented more severe chemosensory anhedonia than the HC group. We also found that chemosensory hedonic capacity was negatively correlated with negative schizotypal traits in the SCT group. Our results suggested that chemosensory anhedonia is an important characteristic of schizophrenia spectrum disorders.

Keywords: schizotypy, schizophrenia, smell, taste, anhedonia

\section{INTRODUCTION}

Anhedonia, the loss or decline of the ability to enjoy pleasure, is an important clinical characteristic of schizophrenia $(1,2)$. It has been consistently reported that schizophrenia patients presented severe physical and social anhedonia (3-5). Similar to the clinical symptoms of schizophrenia but in a weakened form, schizotypy is a group of behavioral traits that indicates the nature of schizotype and shows a high risk for schizophrenia $(2,6)$. More severe anhedonia was also reported in schizotypy individuals according to their performance on the Temporal Experience of Pleasure 
Scale, which means that they experienced relatively less pleasure overall (7). Specifically, according to previous studies, schizotypy individuals presented more severe social and physical anhedonia than healthy controls (5, 7-9). However, few studies have specifically investigated the chemosensory hedonic capacity of schizophrenia patients and schizotypy individuals.

Chemosensory hedonic capacities which refer to the ability to experience pleasure simulated by smell or taste are of great importance to the study of schizophrenia and schizotypy individuals. There is only one synapse between olfactory receptors and the olfactory cortex and there is no thalamic intermediary on olfactory processing, which may provide a direct processing pathway between sensory environment and brain areas $(10,11)$. In addition, there is an overlap in brain regions regulating olfactory function and emotion processing including the orbitofrontal cortex (OFC), amygdala, and hippocampus (12). Therefore, compared with other sensory stimuli, olfaction may be more sensitive in the detection of anhedonia. It has been found that olfactory function was negatively correlated with anhedonia in schizophrenia and schizotypy individuals $(7,9)$. The impairments of olfactory hedonic processing were also reported in schizophrenia (13). Additionally, increased negative symptoms in schizophrenia was related to limited range of olfactory preference ratings $(14,15)$ and increased olfactory anhedonia $(12,16)$.

There are two components of hedonic experience including anticipatory and consummatory pleasure which reflects anticipation for future pleasurable events and reaction to inthe-moment pleasure, respectively (17). Based on these two domains, the Temporal Experience of Pleasure Scale $(18,19)$ and the Anticipatory and Consummatory Interpersonal Pleasure Scale (20) were widely used to assess individual's hedonic capacity. However, none of these scales specifically measure the ability to experience current or noncurrent chemosensory pleasure. To capture these components of anhedonia, Zhao et al. (21) developed the Chemosensory Pleasure Scale (CPS), a chemosensory hedonic measurement to assess smell and taste hedonic capacities. According to previous findings, schizophrenia patients mainly reported diminished anticipatory but not consummatory pleasure (22-25), which has been named the "emotional paradox" of schizophrenia (22). However, previous laboratory studies only investigated the current positive response (i.e., consummatory dimension) toward olfactory stimuli (26), so it is still unclear if schizophrenia patients also present the same "emotional paradox" in the chemosensory hedonic capacities. Therefore, we chose to use the CPS for a further investigation in the chemosensory anticipatory pleasure.

The aims of our study were to use the CPS to: 1) measure the differences in chemosensory hedonic capacities among schizophrenia patients (SCZ), schizotypy individuals (SCT), and healthy controls (HC); and 2) also examine the correlations between the chemosensory hedonic capacity and schizotypal traits or clinical syndromes. We hypothesized that the SCT group would report lower levels of chemosensory anhedonia than the SCZ group but both groups would present higher levels than the HC. Moreover, previous studies found that there was a relationship between the increased olfactory anhedonia and increased negative symptoms in schizophrenia $(12,16)$. Therefore, we also hypothesized that the ability to experience chemosensory pleasure was negatively associated with negative schizotypal traits or syndromes.

\section{METHODS}

\section{Participants}

This study recruited three groups of participants including schizophrenia patients (SCZ), schizotypy individuals (SCT), and healthy controls (HC). Seventy-one schizophrenia patients including 15 first-episode patients (21.13\%) (52 males, 19 females; age range 16 to 31 years, mean $\pm \mathrm{SD}=22.82 \pm 3.82$ years) were recruited from the Guangdong 999 Brain Hospital in Guangzhou, Guangdong, China. All the participants were greater than or equal to 16 years old, and also had greater than or equal to 5 years of education. They were all free from bad cold in recent week, history of nose or throat diseases, brain injury, drug abuse, and other neuropsychiatric disorders according to the oral reports from themselves, their families, or medical records. All the patients were diagnosed by licensed psychiatrists in accordance with the Diagnostic and Statistical Manual of Mental Disorders, 5th Edition (DSM-5) diagnostic criteria for schizophrenia (27). Their clinical symptoms were measured by the Positive and Negative Syndrome Scale (PANSS) (28). Excluding 13 patients without medication records and 2 patients without any antipsychotic medical treatments, a total of 56 patients were taking antipsychotic medications. Dosages of medication were converted into chlorpromazine equivalents (CPZ) (29) (see Table 1).

One hundred and sixty-two individuals with schizotypy (60 males, 102 females; age range 17 to 21 years, mean $\pm \mathrm{SD}=18.27 \pm$ 0.76 years) and 182 controls ( 74 males, 108 females; age range 16 to 21 years, mean $\pm \mathrm{SD}=18.27 \pm 0.85$ years) were recruited from the Southern Medical University in Guangzhou, Guangdong, China. They were selected from a sample of 1,589 college students according to their performance on the Schizotypal Personality Questionnaire (SPQ) $(30,31)$. Participants scoring at the top and bottom $10 \%$ were considered as the SCT group (scored higher than 41) and the HC group (scored lower than 10), respectively (8, 9, 31). Base on their self-reports, they were all free from bad cold in recent week, history of nose or throat diseases, brain injury, drug abuse, and neuropsychiatric disorders.

Our study was approved by the Ethics Committees of Southern Medical University and Guangdong 999 Brain Hospital. All the participants were provided informed consent before our study began.

\section{Measures}

\section{Chemosensory Hedonic Traits}

The Chemosensory Pleasure Scale (CPS) is a self-rating scale to assess individual's abilities to enjoy smell and taste stimulation (21). It consists of 12 items capturing 3 factors: food, imagination, and nature which refers to "consummatory," 
TABLE 1 | Demographics and clinical characteristics of the participants.

\begin{tabular}{|c|c|c|c|c|c|c|c|c|}
\hline & \multicolumn{2}{|c|}{$\begin{array}{l}\text { Schizophrenia group } \\
\qquad(n=71)\end{array}$} & \multicolumn{2}{|c|}{$\begin{array}{l}\text { Schizotypy group } \\
\qquad(n=162)\end{array}$} & \multicolumn{2}{|c|}{$\begin{array}{l}\text { Healthy controls } \\
\qquad(n=182)\end{array}$} & \multirow[t]{2}{*}{$F / \chi^{2}$} & \multirow[t]{2}{*}{ Post-hoc } \\
\hline & Mean & $S D$ & Mean & $S D$ & Mean & $S D$ & & \\
\hline Age (years) & 22.82 & 3.82 & 18.27 & 0.76 & 18.27 & 0.85 & $94.77^{\star \star \star}$ & $\mathrm{HC}, \mathrm{SCT}<\mathrm{SCZ}$ \\
\hline $\operatorname{Sex}(F: M)$ & $19: 52$ & & $102: 60$ & & $108: 74$ & & $28.43^{\star \star \star}$ & $\mathrm{HC}, \mathrm{SCT}>\mathrm{SCZ}$ \\
\hline Education (years) & 9.79 & 2.76 & - & - & - & - & - & - \\
\hline PANSS & & & & & & & - & - \\
\hline Positive symptoms & 18.77 & 6.09 & - & - & - & - & - & - \\
\hline Negative symptoms & 23.41 & 7.21 & - & - & - & - & - & - \\
\hline General psychopathology & 44.19 & 12.04 & - & - & - & - & - & - \\
\hline Chlorpromazine equivalents(mg/day) & 397.71 & 228.07 & - & - & - & - & - & - \\
\hline
\end{tabular}

F, female; M, male; PANSS, Positive and Negative Syndrome Scale; SD, standard deviation. ${ }^{* * *} p<0.001$.

"anticipatory," and "purely olfactory" dimensions, reflecting the hedonic capacities of eating, anticipating food, and smelling natural scents, respectively. Participants were required to report their pleasant experience of smell and taste based on the 6-point Likert scale (from 1 "very false for me" to 6 "very true for me"). A lower score on the CPS indicated more severe chemosensory anhedonia. The Cronbach's $\alpha$ coefficient was 0.93 and the test-retest reliabilities of the CPS was 0.73 .

\section{Schizotypal Traits}

The Schizotypal Personality Questionnaire (SPQ) is a self-rating scale for schizotypal personality disorder according to the DSMIII-R criteria (31). It contains 74 items capturing all nine schizotypal traits. It has a high internal reliability $(0.91)$ and test-retest reliability (0.82). It can be used for screening for schizotypal personality disorder across normal people, and also for studying the correlation of individual schizotypal traits. In our study, we used the Chinese version of SPQ (30). It is a threefactor model including the cognitive-perceptual (also referred to as positive schizotypy), the interpersonal (also referred to as negative schizotypy), and the disorganized factors.

\section{Data Analysis}

Data analysis was conducted with SPSS version 22 (SPSS Inc, Chicago, IL, USA). Descriptive statistics were used to characterize the SCT, SCZ, and HC groups separately. The normal distribution was assessed using Shapiro-Wilk normality test. Because of the non-normal distribution of the CPS data, the non-parametric Kruskal-Wallis test followed by DunnBonferroni test for post hoc comparisons were used to analyze the group differences on CPS total and subscale scores. In addition, the effect size estimates of the Kruskal-Wallis test were calculated using the eta squared $\left(n^{2}\right)$ measure. The formula of the $\mathrm{n}^{2}$ estimates using $\mathrm{H}$ statistic was as follows (32):

$$
\mathrm{n}_{H}^{2}=\frac{H-k+1}{n-k}
$$

As for the formula, the $\mathrm{H}$ value was the statistic of the Kruskal-Wallis test. The $\mathrm{k}$ and $\mathrm{n}$ value were the number of groups and the total number of participants, respectively.

Furthermore, we also calculated the correlations between the CPS and SPQ, PANSS, dose of medications as well as duration of illness. Pearson's correlation analysis was used if the data was normally distributed, otherwise Spearman's correlation analysis was conducted. The multiple correlation analyses were also corrected with the Bonferroni correction.

\section{RESULTS}

There were significant differences in age $[F(2,412)=94.77, p<$ $0.001]$ and $\operatorname{sex}\left(\chi^{2}=28.43, p<0.001\right)$ among the three groups. The post-hoc comparison showed that the average age of SCZ group was significantly older than that of SCT group and HC group, while the SCT group and HC group had higher sex ratio than the SCZ group (see Table 1).

As shown in Table 2, the three groups differed significantly in the CPS total and three subscales scores (total: $H=86.824, p<$ $0.001, \mathrm{n}^{2}=0.21$; food: $H=37.612, p<0.001, \mathrm{n}^{2}=0.09$; imagination: $H=120.530, p<0.001, \mathrm{n}^{2}=0.29$; nature:

TABLE 2 | Chemosensory hedonic traits.

\begin{tabular}{|c|c|c|c|c|c|c|}
\hline & $\begin{array}{l}\text { Schizophrenia patients } \\
\qquad(\mathrm{n}=71)\end{array}$ & $\begin{array}{l}\text { Schizotypy group } \\
\text { ( } n=162)\end{array}$ & $\begin{array}{l}\text { Healthy controls } \\
\qquad(n=182)\end{array}$ & $H$ & $\begin{array}{l}\text { Effect size } \\
\qquad\left(\eta^{2}\right)\end{array}$ & Pos-hoc \\
\hline & $\begin{array}{l}\text { Median } \\
\text { (Min-max) }\end{array}$ & $\begin{array}{l}\text { Median } \\
\text { (Min-max) }\end{array}$ & $\begin{array}{l}\text { Median } \\
\text { (Min-max) }\end{array}$ & & & \\
\hline CPS total & $45(12-70)$ & 59 (12-72) & $60(30-72)$ & $86.824^{\star \star \star}$ & 0.21 & $\mathrm{HC}, \mathrm{SCT}>\mathrm{SCZ}$ \\
\hline Food & $20(5-30)$ & $24(5-30)$ & $25(12-30)$ & $37.612^{\star \star \star}$ & 0.09 & $\mathrm{HC}>\mathrm{SCT}>\mathrm{SCZ}$ \\
\hline Imagination & $13(4-23)$ & $20(4-24)$ & $21(11-24)$ & $120.530^{\star \star *}$ & 0.29 & $\mathrm{HC}, \mathrm{SCT}>\mathrm{SCZ}$ \\
\hline
\end{tabular}

min, minimum score; max, maximum score; ${ }^{* * *} p<0.001$. 
$\left.H=32.385, p<0.001, \mathrm{n}^{2}=0.07\right)$. Post-hoc comparisons found that the SCZ group presented significantly lower scores on the CPS and all the subscales than the remaining two groups. Besides, compared with the HC group, the SCT group showed significantly lower scores in the food subscale, but these two groups did not differ significantly in the CPS total scores, imagination and nature subscales.

In the SCT group, the positive correlations were observed between the cognitive-perceptual dimension of SPQ and the CPS total score as well as the three subscales scores (total: $r=0.21, p<$ 0.01; food: $r=0.22, p<0.01$; imagination: $r=0.16, p<0.05$; nature: $r=0.19, p<0.05)$. However, after Bonferroni correction, only the food subscale was significantly correlated with the cognitive-perceptual dimension $(p<0.006)$. The interpersonal dimension of SPQ was negatively correlated with the CPS total score (see Figure 1), the food and imagination subscales scores (total: $r=-0.23, p<0.01$; food: $r=-0.25, p<0.01$; imagination: $r=-0.23, p<0.01)$, but not with the nature subscale $(r=-0.15$, $p>0.05)$, and the correlations were still significant after Bonferroni correction $(p<0.006)$ (see Table 3). In the SCZ group, the negative syndromes subscale of PANSS had a significantly negative correlation with the food subscale of CPS $(r=-0.25, p<0.05)$, but this correlation was not found after Bonferroni correction $(p>0.007)$. Besides, there were no significant correlations between the negative syndrome subscale of PANSS and the CPS total score, the imagination or nature subscales scores ( $p$ s > 0.05). The relationship between the CPS and the positive syndromes or general psychopathology of PANSS was not statistically significant ( $p$ s $>0.05$ ) (see Table 4). Moreover, the CPS and all three subscales were not significantly correlated with the $\mathrm{CPZ}$ equivalence or duration of illness $(p s>0.05)$.

\section{DISCUSSION}

This is the first study that directly used self-reported chemosensory pleasure scale to compare chemosensory hedonic capacity in schizophrenia patients, schizotypy individuals, and healthy controls. We found that the SCZ group presented more severe chemosensory anhedonia than

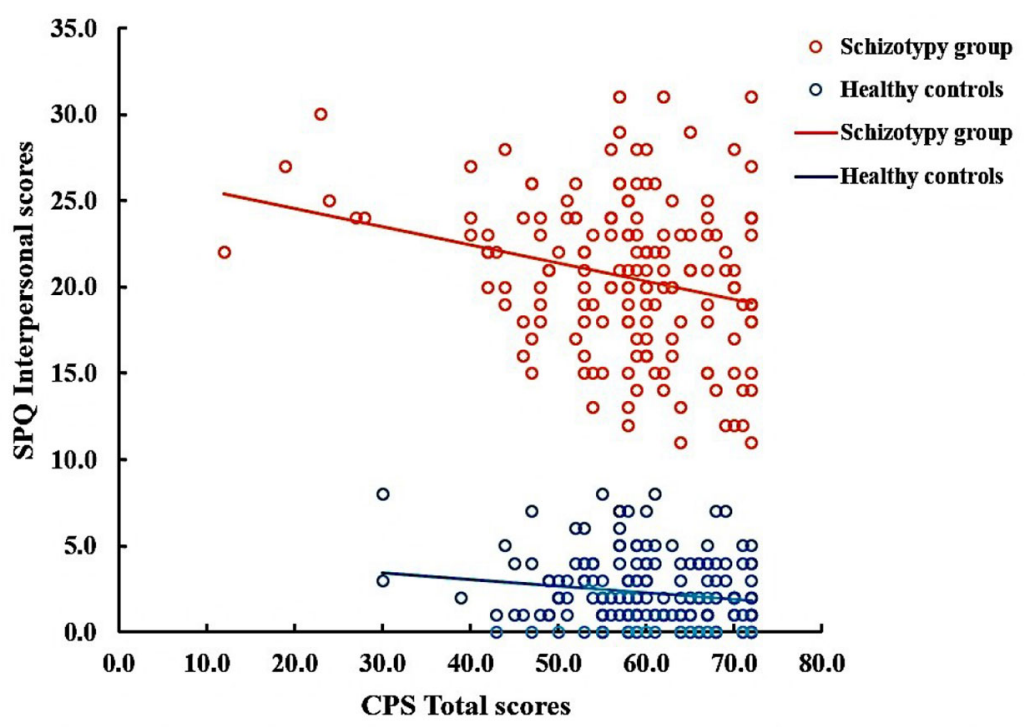

FIGURE 1 | Correlation between Chemosensory Pleasure Scale (CPS) total scores and Schizotypal Personality Questionnaire (SPQ) Interpersonal Scores in schizotypy group $(r=-0.23, p<0.01)$ and healthy controls $(r=-0.14, p=0.063)$.

TABLE 3 | Correlations between Chemosensory Pleasure Scale (CPS) and Schizotypal Personality Questionnaire (SPQ) scores in schizotypy.

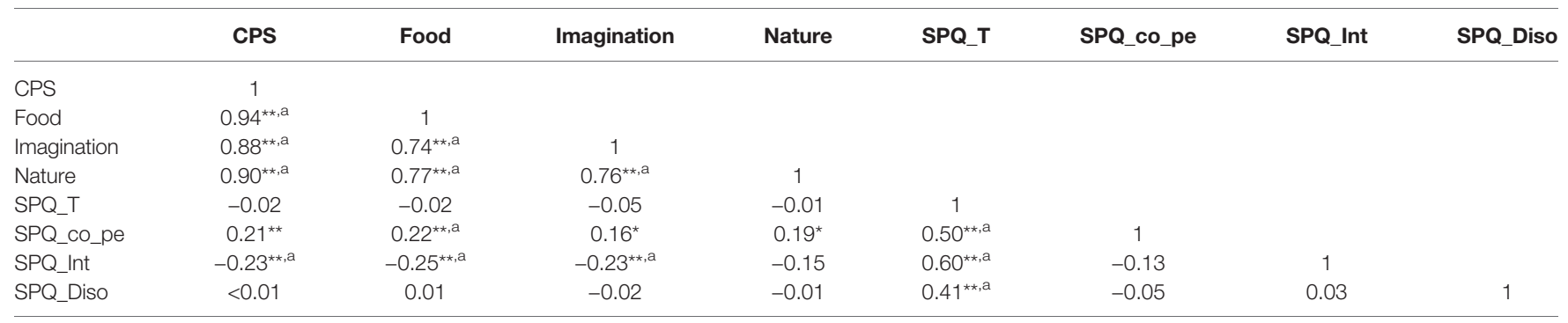

CPS, Chemosensory Pleasure Scale; $S P Q \_t$, Schizotypal Personality Questionnaire total score; SPQ co_pe, SPQ cognitive-perceptual score; SPQ_Int, SPQ interpersonal score; $S P Q \_$Diso, SPQ disorganized score; * $p<0.05 ;{ }^{* *} p<0.01,{ }^{a} p<0.006$. 
TABLE 4 | Correlations between Chemosensory Pleasure Scale (CPS) and Positive and Negative Syndrome Scale (PANSS) in schizophrenia patients.

\begin{tabular}{|c|c|c|c|c|c|c|c|}
\hline & CPS & Food & Imagination & Nature & PANSS_P & PANSS_N & PANSS_G \\
\hline CPS & 1 & & & & & & \\
\hline Food & $0.85^{\star \star, b}$ & 1 & & & & & \\
\hline Imagination & $0.83^{\star \star, b}$ & $0.54^{\star \star, b}$ & 1 & & & & \\
\hline Nature & $0.72^{\star \star, b}$ & $0.46^{\star \star, b}$ & $0.40^{\star \star, b}$ & 1 & & & \\
\hline PANSS_P & 0.05 & -0.05 & 0.09 & 0.09 & 1 & & \\
\hline PANSS_N & -0.18 & $-0.25^{\star}$ & -0.06 & -0.14 & 0.16 & 1 & \\
\hline PANSS_G & 0.06 & -0.17 & 0.24 & 0.09 & $0.51^{\star \star, b}$ & $0.51^{\star \star, \mathrm{b}}$ & 1 \\
\hline
\end{tabular}

CPS, Chemosensory Pleasure Scale; PANSS, Positive and Negative Syndrome Scale; PANSS_P, PANSS positive syndromes score; PANSS_N, PANSS negative syndromes score; PANSS_G, PANSS general psychopathology score; ${ }^{*} p<0.05 ;{ }^{* *} p<0.01,{ }^{b} p<0.007$.

the SCT group, and the SCT group presented more severe chemosensory anhedonia than the HC group. Additionally, chemosensory hedonic capacity was negatively correlated with negative schizotypal trait in SCT group.

Our finding of less pleasure from chemosensory stimulation in the SCZ group is consistent with previous neurobehavioral studies, which reported that SCZ patients having higher level of olfactory anhedonia than HC $(12,33)$. Moberg et al. (13) also found that hedonic odor processing was disrupted in schizophrenia patients. Moreover, we found that the schizophrenia patients differed from healthy controls in reports of current and noncurrent olfactory pleasure. They showed impairments in anticipatory and consummatory hedonic capacities. This finding was inconsistent with the previous findings that schizophrenia patients mainly reported intact consummatory but diminished anticipatory pleasure (22-25). Furthermore, schizophrenia patients reported the same levels of in-the-moment positive emotion as the healthy controls when processing laboratory stimuli $(26,34)$. The inconsistency of the results may further prove the uniqueness of olfaction. The overlapping brain regions between olfactory and emotion processing (12) and olfactory evolutionary significance in eating (35), selecting mates (36), and warning danger or threat (37) might make olfaction particularly sensitive to hedonic perception. For the individuals with schizotypy, previous studies consistently showed that they had significantly higher physical and social anhedonia (5, 7-9). Our study further investigated the chemosensory anhedonia in individuals with schizotypy. Just as the results of the studies on social and physical anhedonia, the SCT group showed more severe chemosensory anhedonia than the HC group. Our findings suggested that decreased chemosensory hedonic capacity may be an important trait marker in schizophrenia spectrum disorders.

In previous studies, it has been repeatedly found that negative symptoms were associated with olfactory deficits in schizophrenia patients $(7,38-41)$. In our study, although there were no significant differences between the SCT and HC on the CPS total, imagination, and nature subscales scores, we found that negative schizotypal trait was negatively correlated with the pleasant experience of smell and taste. This is consistent with previous studies which also reported that more severe negative symptoms were associated with a tendency to poorly evaluate hedonic experience of smell $(12,16,42)$. It may suggest that chemosensory anhedonia was an important feature of negative schizotypal trait, but not positive schizotypal trait, which supports the idea that negative and positive schizotypy represent discrete dimensions (43). In the future, it may be important to further distinguish positive and negative schizotypy populations and compare their chemosensory hedonic capacities. Moreover, the food subscale was negatively correlated with the PANSS negative symptoms in the SCZ group, but this correlation was not found after Bonferroni correction. Besides, the significant negative correlations between negative symptoms and the CPS or the other two subscales scores were not observed in our study. The possible reason is that the clinical symptoms in our study were only measured by a single scale (i.e., PANSS), and the PANSS contains no items to measure anhedonia, unlike the Scale for the Assessment of Negative Symptoms (SANS) (44). The SANS and the Clinical Assessment Interview for Negative Symptoms (CAINS) (45) are sensitive and specific clinical measurements of negative symptoms in schizophrenia. In future studies, the SANS or CAINS should be used to measure the negative symptoms of schizophrenia in more details.

There were several limitations in this study. The age and sex of the SCZ group did not match well with the other two groups (the SCT and HC groups), so further research is needed to reduce the age and sex bias. Nevertheless, the SCT high-risk group showed higher levels of chemosensory anhedonia than the demographics well-matched HC group. In addition, participants did not rate the pleasantness in relation to the smelling of actual odors and or the eating of actual foods in our study, which should also be assessed in the future.

In summary, both patients with schizophrenia and schizotypy individuals showed more severe chemosensory anhedonia than health controls. Besides, the results also showed that chemosensory anhedonia was associated with negative schizotypal traits. Our findings further suggest that the decreased chemosensory hedonic capacity may be an important trait marker in schizophrenia spectrum disorders.

\section{DATA AVAILABILITY STATEMENT}

The datasets generated for this study are available on request to the corresponding authors. 


\section{ETHICS STATEMENT}

The studies involving human participants were reviewed and approved by Ethics Committees of Southern Medical University and Guangdong 999 Brain Hospital. Written informed consent to participate in this study was provided by the participants' legal guardian/next of kin.

\section{AUTHOR CONTRIBUTIONS}

Z-LL and G-JH contributed equally to the study: they collected and analyzed the data, and wrote the first draft of the manuscript. Z-TL, S-BL and Y-LW collected and analyzed the data. J-FW collected and analyzed the data, and wrote the first draft of the manuscript. J-BZ and TH interpreted the data, and gave the comment to the first draft of the manuscript. L-QZ generated the

\section{REFERENCES}

1. Horan WP, Kring AM, Blanchard JJ. Anhedonia in schizophrenia: a review of assessment strategies. Schizophr Bull (2006) 32(2):259-73. doi: 10.1093/ schbul/sbj009

2. Meehl PE. Schizotaxia, schizotypy, schizophrenia. Am Psychol (1962) 17 (12):827. doi: 10.1037/h0041029

3. Blanchard JJ, Mueser KT, Bellack AS. Anhedonia, positive and negative affect, and social functioning in schizophrenia. Schizophr Bull (1998) 24(3):413-24. doi: 10.1093/oxfordjournals.schbul.a033336

4. Burbridge JA, Barch DM. Anhedonia and the experience of emotion in individuals with schizophrenia. J Abnorm Psychol (2007) 116(1):30-42. doi: 10.1037/0021-843X.116.1.30

5. Wang Y, Lui SS, Zou LQ, Zhang Q, Zhao Q, Yan C, et al. Individuals with psychometric schizotypy show similar social but not physical anhedonia to patients with schizophrenia. Psychiatry Res (2014) 216(2):161-7. doi: 10.1016/ j.psychres.2014.02.017

6. Meehl PE. Toward an integrated theory of schizotaxia, schizotypy, and schizophrenia. J Pers Disord (1990) 4(1):1-99. doi: 10.1521/pedi.1990.4.1.1

7. Zou LQ, Zhou HY, Lui SSY, Wang Y, Wang Y, Gan J, et al. Olfactory identification deficit and its relationship with hedonic traits in patients with first-episode schizophrenia and individuals with schizotypy. Prog Neuropsychopharmacol Biol Psychiatry (2018) 83:137-41. doi: 10.1016/ j.pnpbp.2018.01.014

8. Chan RC, Wang Y, Yan C, Zhao Q, McGrath J, Hsi X, et al. A study of trait anhedonia in non-clinical Chinese samples: evidence from the Chapman Scales for Physical and Social Anhedonia. PloS One (2012) 7(4):e34275. doi: 10.1371/journal.pone.0034275

9. Zou LQ, Geng FL, Liu WH, Wei XH, Jiang XQ, Wang Y, et al. The neural basis of olfactory function and its relationship with anhedonia in individuals with schizotypy: An exploratory study. Psychiatry Res (2015) 234(2):202-7. doi: 10.1016/j.pscychresns.2015.09.011

10. Gottfried JA. Smell: central nervous processing. Adv oto-rhino-laryngology (2006) 63:44-69. doi: 10.1159/000093750

11. Patel RM, Pinto JM. Olfaction: anatomy, physiology, and disease. Clin Anat (2014) 27(1):54-60. doi: 10.1002/ca.22338

12. Kamath V, Moberg PJ, Kohler CG, Gur RE, Turetsky BI. Odor hedonic capacity and anhedonia in schizophrenia and unaffected first-degree relatives of schizophrenia patients. Schizophr Bull (2013) 39(1):59-67. doi: 10.1093/ schbul/sbr050

13. Moberg PJ, Kamath V, Marchetto DM, Calkins ME, Doty RL, Hahn CG, et al. Meta-analysis of olfactory function in schizophrenia, first-degree family members, and youths at-risk for psychosis. Schizophr Bull (2014) 40(1):509. doi: $10.1093 /$ schbul/sbt049 idea, designed the study, interpreted the data, and wrote the first draft of the manuscript.

\section{ACKNOWLEDGMENTS}

This work was supported by the National Natural Science Foundation of China [grand number: 31700963], the Natural Science Foundation of Guangdong Province, China [grand number: 2017A030310069], and the Medical Science and Technology Foundation of Guangdong Province, China [grant number: 201906546]. These funding agents had no further role in the study design; in collecting, analyzing or interpreting the data; in writing the manuscript; or in the decision to submit the paper for publication. The authors thank the Mental Health Center of Southern Medical University for providing support during the data collection stage of our study.

14. Cumming AG, Matthews NL, Park S. Olfactory identification and preference in bipolar disorder and schizophrenia. Eur Arch Psychiatry Clin Neurosci (2011) 261(4):251-9. doi: 10.1007/s00406-010-0145-7

15. Doop ML, Park S. On knowing and judging smells: identification and hedonic judgment of odors in schizophrenia. Schizophr Res (2006) 81(2-3):317-9. doi: 10.1016/j.schres.2005.08.006

16. Strauss GP, Allen DN, Ross SA, Duke LA, Schwartz J. Olfactory hedonic judgment in patients with deficit syndrome schizophrenia. Schizophr Bull (2010) 36(4):860-8. doi: 10.1093/schbul/sbn178

17. Kring AM, Elis O. Emotion deficits in people with schizophrenia. Annu Rev Clin Psychol (2013) 9:409-33. doi: 10.1146/annurev-clinpsy-050212-185538

18. Chan RC, Shi YF, Lai MK, Wang YN, Wang Y, Kring AM. The Temporal Experience of Pleasure Scale (TEPS): exploration and confirmation of factor structure in a healthy Chinese sample. PloS One (2012) 7(4):e35352. doi: 10.1371/journal.pone.0035352

19. Gard DE, Gard MG, Kring AM, John OP. Anticipatory and consummatory components of the experience of pleasure: A scale development study. J Res Pers (2006) 40(6):1086-102. doi: 10.1016/j.jrp.2005.11.001

20. Gooding DC, Pflum MJ. The assessment of interpersonal pleasure: introduction of the Anticipatory and Consummatory Interpersonal Pleasure Scale (ACIPS) and preliminary findings. Psychiatry Res (2014) 215(1):237-43. doi: 10.1016/j.psychres.2013.10.012

21. Zhao JB, Wang YL, Ma QW, Zhao JB, Zhang XY, Zou LQ. The Chemosensory Pleasure Scale: A New Assessment for Measuring Hedonic Smell and Taste Capacities. Chem Senses (2019) 44(7):457-64. doi: 10.1093/chemse/ bjz040

22. Strauss GP. The emotion paradox of anhedonia in schizophrenia: or is it? Schizophr Bull (2013) 39(2):247-50. doi: 10.1093/schbul/sbs192

23. Gard DE, Kring AM, Gard MG, Horan WP, Green MF. Anhedonia in schizophrenia: distinctions between anticipatory and consummatory pleasure. Schizophr Res (2007) 93(1-3):253-60. doi: 10.1016/ j.schres.2007.03.008

24. Yan C, Lui SSY, Zou LQ, Wang CY, Zhou FC, Cheung EFC, et al. Anticipatory pleasure for future rewards is attenuated in patients with schizophrenia but not in individuals with schizotypal traits. Schizophr Res (2019) 206:118-26. doi: 10.1016/j.schres.2018.12.003

25. Favrod J, Ernst F, Giuliani F, Bonsack C. [Validation of the Temporal Experience of Pleasure Scale (TEPS) in a French-speaking environment]. L'Encephale (2009) 35(3):241-8. doi: 10.1016/j.encep.2008.02.013

26. Cohen AS, Minor KS. Emotional experience in patients with schizophrenia revisited: meta-analysis of laboratory studies. Schizophr Bull (2010) 36 (1):143-50. doi: 10.1093/schbul/sbn061

27. American Psychiatric Association. Diagnostic and statistical manual of mental disorders (DSM-5 $\left.{ }^{\circledR}\right)$ Washington, DC. (2013). 
28. Kay SR, Fiszbein A, Opler LA. The positive and negative syndrome scale (PANSS) for schizophrenia. Schizophr Bull (1987) 13(2):261-76. doi: 10.1093/ schbul/13.2.261

29. Woods SW. Chlorpromazine equivalent doses for the newer atypical antipsychotics. J Clin Psychiatry (2003) 64(6):663-7. doi: 10.4088/ jcp.v64n0607

30. Chen WJ, Hsiao CK, Lin CC. Schizotypy in community samples: the threefactor structure and correlation with sustained attention. J Abnorm Psychol (1997) 106(4):649-54. doi: 10.1037//0021-843x.106.4.649

31. Raine A. The SPQ: a scale for the assessment of schizotypal personality based on DSM-III-R criteria. Schizophr Bull (1991) 17(4):555-64. doi: 10.1093/schbul/17.4.555

32. Tomczak M, Tomczak E. The need to report effect size estimates revisited. An overview of some recommended measures of effect size. Trends Sport Sci (2014) 21(1):19-25.

33. Plailly J, d'Amato T, Saoud M, Royet JP. Left temporo-limbic and orbital dysfunction in schizophrenia during odor familiarity and hedonicity judgments. Neuroimage (2006) 29(1):302-13. doi: 10.1016/j.neuroimage.2005.06.056

34. Yan C, Cao Y, Zhang Y, Song LL, Cheung EF, Chan RC. Trait and state positive emotional experience in schizophrenia: a meta-analysis. PloS One (2012) 7(7):e40672. doi: 10.1371/journal.pone.0040672

35. Luisier AC, Petitpierre G, Ferdenzi C, Clerc Berod A, Giboreau A, Rouby C, et al. Odor Perception in Children with Autism Spectrum Disorder and its Relationship to Food Neophobia. Front Psychol (2015) 6:1830. doi: 10.3389/fpsyg.2015.01830

36. Mahmut MK, Croy I. The role of body odors and olfactory ability in the initiation, maintenance and breakdown of romantic relationships - A review. Physiol Behav (2019) 207:179-84. doi: 10.1016/j.physbeh.2019.05.003

37. Santos DV, Reiter ER, DiNardo LJ, Costanzo RM. Hazardous events associated with impaired olfactory function. Arch otolaryngology-head Neck Surg (2004) 130(3):317-9. doi: 10.1001/archotol.130.3.317

38. Brewer WJ, Pantelis C, Anderson V, Velakoulis D, Singh B, Copolov DL, et al. Stability of olfactory identification deficits in neuroleptic-naive patients with first-episode psychosis. Am J Psychiatry (2001) 158(1):107-15. doi: 10.1176/ appi.ajp.158.1.107

39. Malaspina D, Coleman E, Goetz RR, Harkavy-Friedman J, Corcoran C, Amador $\mathrm{X}$, et al. Odor identification, eye tracking and deficit syndrome schizophrenia. Biol Psychiatry (2002) 51(10):809-15. doi: 10.1016/s0006-3223 (01)01319-1

40. Corcoran C, Whitaker A, Coleman E, Fried J, Feldman J, Goudsmit N, et al. Olfactory deficits, cognition and negative symptoms in early onset psychosis. Schizophr Res (2005) 80(2-3):283-93. doi: 10.1016/j.schres.2005.07.028

41. Ishizuka K, Tajinda K, Colantuoni C, Morita M, Winicki J, Le C, et al. Negative symptoms of schizophrenia correlate with impairment on the University of Pennsylvania smell identification test. Neurosci Res (2010) 66 (1):106-10. doi: 10.1016/j.neures.2009.10.001

42. Auster TL, Cohen AS, Callaway DA, Brown LA. Objective and subjective olfaction across the schizophrenia spectrum. Psychiatry (2014) 77(1):57-66. doi: 10.1521/psyc.2014.77.1.57

43. Dinn WM, Harris CL, Aycicegi A, Greene P, Andover MS. Positive and negative schizotypy in a student sample: neurocognitive and clinical correlates. Schizophr Res (2002) 56(1-2):171-85. doi: 10.1016/S0920-9964 (01)00230-4

44. Andreasen NC. The Scale for the Assessment of Negative Symptoms (SANS): Conceptual and Theoretical Foundations. Brit J Psychiat (1981) 155(S7):4952. doi: $10.1192 / \$ 0007125000291496$

45. Kring AM, Gur RE, Blanchard JJ, Horan WP, Reise SP. The Clinical Assessment Interview for Negative Symptoms (CAINS): final development and validation. Am J Psychiatry (2013) 170(2):165-72. doi: 10.1176/ appi.ajp.2012.12010109

Conflict of Interest: The authors declare that the research was conducted in the absence of any commercial or financial relationships that could be construed as a potential conflict of interest.

Copyright $\odot 2020$ Li, Huang, Li, Li, Wang, Zhao, Wen, Hummel and Zou. This is an open-access article distributed under the terms of the Creative Commons Attribution License (CC BY). The use, distribution or reproduction in other forums is permitted, provided the original author(s) and the copyright owner(s) are credited and that the original publication in this journal is cited, in accordance with accepted academic practice. No use, distribution or reproduction is permitted which does not comply with these terms. 